\title{
Atypical presentation of COVID-19 in hospitalised older adults
}

\author{
Jasmine Ming Gan ${ }^{1} \cdot$ Jason Kho ${ }^{1}$ - Michelle Akhunbay-Fudge ${ }^{1} \cdot$ Hwei Ming Choo ${ }^{1} \cdot$ Melanie Wright $^{1} \cdot$ Farzana Batt $^{1}$. \\ Amit K. J. Mandal ${ }^{1}$ (D) $\cdot$ Rahul Chauhan $^{1} \cdot$ Constantinos G. Missouris ${ }^{1,2}$
}

Received: 19 July 2020 / Accepted: 17 September 2020 / Published online: 21 September 2020

(C) Royal Academy of Medicine in Ireland 2020

\begin{abstract}
Background It is increasingly recognised that older patients may not present with typical symptoms of COVID-19. Aims This study aims to evaluate the incidence, characteristics and clinical outcome of older adults with atypical presentations of COVID-19.

Methods A retrospective analysis of adults $\geq 65$ years with confirmed COVID-19 admitted to our institution between 1 March and 24 April 2020 was performed. Patients were categorised into typical or atypical groups based on primary presenting complaint in the community.

Results One hundred twenty-two patients (mean age $81 \pm 8$ years; 62 male) were included. Seventy-three (60\%) were categorised into the typical group and $49(40 \%)$ into the atypical group. In the atypical group, common presenting complaints were fall in 18 $(36 \%)$, reduced mobility or generalised weakness in $18(36 \%)$ and delirium in $11(22 \%)$. Further assessment by paramedics and on admission found $32(65 \%)$ to have typical features of COVID-19, fever being the most common, and 22 (44\%) were hypoxic. This subset had worse outcomes than those in the typical group with a mortality rate of $50 \%$ versus $38 \%$, respectively, although this was not statistically significant $(P=0.27)$. No significant difference in mortality or length of hospital stay between the groups was demonstrated.

Conclusion Older patients with atypical presentation of COVID-19 in the community are equally susceptible to poor outcomes. Early detection may improve outcomes and limit community transmission. Primary care practitioners should be vigilant and consider prompt onward referral.
\end{abstract}

Keywords Atypical symptoms · Coronavirus · COVID-19 · Older adults

\section{Introduction}

The first case of coronavirus disease 2019 (COVID-19) in the UK was reported on 30 January 2020, and early transmission within the country became evident during February. Since then the rate of infection has increased exponentially in unison with the rate of death, claiming over 42,000 lives in the UK alone [1]. COVID-19 is primarily a respiratory disease, and usual symptoms reported include pyrexia, dry cough, dyspnoea, anosmia and ageusia $[2,3]$. A small percentage of

Amit K. J. Mandal

amit.mandal@nhs.net

1 Department of Medicine, Wexham Park Hospital, Frimley Health NHS Foundation Trust, Wexham Street, Slough, UK

2 University of Cyprus Medical School, Nicosia, Cyprus patients develop severe disease complicated by respiratory failure and require ventilatory support [3].

Evidence suggests that adults aged 50 years and over with multiple comorbidities are more susceptible to severe COVID-19 and are at higher risk of dying [4, 5]. It is wellrecognised that older adults in acute illness states commonly present with atypical symptoms of falls, reduced mobility or delirium [6], and it is apparent that this is the case with COVID-19. The diagnosis can be even more demanding if nasopharyngeal swabs for severe acute respiratory syndrome coronavirus-2 (SARS-CoV-2) are not readily accessible in primary and community care settings. Resultantly, a significant proportion of deaths attributed to COVID-19 in the UK are being reported in residential care homes [7]. Social distancing and lockdown measures introduced by governmental bodies worldwide, including that of the UK, might not be favourable for an older population because of the lack of social support and the unavailability of COVID-19 testing in the community. This could hinder timely treatment and admission 
to hospital where necessary. The British Geriatrics Society has therefore highlighted the need for clinicians to have an index of suspicion for atypical presentations of COVID-19 in the older population [8].

We set out to evaluate the incidence, characteristics and the clinical outcome of patients aged 65 years or older with atypical presentations of COVID-19 in the community.

\section{Methods}

\section{Study design and patient population}

A retrospective analysis of all adults $\geq 65$ years with confirmed COVID-19 admitted to an acute geriatric medicine unit within a National Health Service (NHS) trust between 1 March and 24 April 2020 was performed. During the study period, nasopharyngeal swabs were only performed if there was a clinical suspicion for COVID-19 as per national guidelines. However, chest X-rays were performed routinely on all admissions in line with local trust protocol.

A diagnosis of COVID-19 in our cohort was confirmed with positive real-time reverse transcriptase-polymerase chain reaction (RT-PCR) assay of nasopharyngeal swabs. When there was a strong clinical suspicion of COVID-19 among patients with negative RT-PCR assay, a radiological diagnosis with either chest X-ray or computed tomography (CT) imaging of the chest was utilised. Radiological evidence of COVID-19 included bilateral peripheral infiltrates/groundglass opacities, airspace opacification, traction bronchiectasis, inter/intralobular septal thickening and organising pneumonia.

Patients were included for analysis if the reason for admission to hospital was likely precipitated by active COVID-19 based on medical documentation. This was analysed by two independent observers to mitigate selection bias. Patient data including demographics, presenting complaints, comorbidities, Rockwood clinical frailty score (CFS), pre-existing "Do Not Attempt Resuscitation" document, vital signs on admission and inpatient investigations were extracted from medical notes and the local hospital electronic database. Hypoxia on admission was defined as room air oxygen saturations on pulse oximeter of $<94 \%$ and a new oxygen requirement which was adjusted to $<88 \%$ for patients with chronic type 2 respiratory failure. Laboratory investigations analysed were complete blood count, C-reactive protein (CRP), coagulation profile, creatine kinase $(\mathrm{CK})$, serum biochemistry (including markers of liver and renal function), lactate dehydrogenase (LDH), serum ferritin, serum Troponin-T and D-dimer.

Comorbidities recorded and analysed in our study included dementia, hypertension, coronary artery disease, congestive heart failure, chronic obstructive pulmonary disease, asthma, type 2 diabetes mellitus, chronic kidney disease and immunosuppressive treatment. We also documented patients' residential situation, i.e. living at home or in a community institution (residential home, care home or nursing home). Patient outcome including length of stay, ventilatory support, intensive care admission and mortality were analysed.

Patients with suspected nosocomial COVID-19 were excluded from the study, with a cut-off inpatient hospital stay of 5 days.

Patients were categorised into typical and atypical groups based on their primary presenting complaint in the community. Typical presentations of COVID-19 were defined according to the guidelines presented by the Regional Geriatric Program of Toronto-fever, dry cough and dyspnoea [2]. The absence of these symptoms and the presence of other unusual symptoms of COVID-19 such as delirium, falls, mobility issues, lethargy, weakness, reduced oral intake, diarrhoea, dizziness, headache, sore throat, vomiting, reduced conscious levels and chest pain were classified as atypical presentations.

\section{Statistical analysis}

All data were analysed using SPSS software version 26 . Categorical variables were presented as frequencies and percentages. Normally distributed data were expressed as mean $\underline{\underline{ }}$ standard deviation, while non-normally distributed data were expressed as median \pm interquartile range. Tests of normality (Kolmogorov-Smirnov and Shapiro Wilk tests) were used to confirm normality of continuous variables. Student's $t$ test was performed to compare normally distributed data, while Mann-Whitney $U$ test was performed to compare nonnormally distributed data. Chi-square test was performed to compare categorical variables between groups: gender, comorbidities and clinical frailty scores. $P$ value of $<0.05$ (two-tailed test) was considered to be statistically significant.

\section{Results}

One hundred twenty-two patients met the inclusion criteria for our study. Patient demographics and characteristics are summarised in Table 1. One hundred six patients $(87 \%)$ had positive nasopharyngeal swabs for SARS-CoV-2, while 16 patients $(13 \%)$ with negative swabs were diagnosed using chest imaging modalities. Out of 122 patients (mean age 81 \pm 8 years, 62 male), $73(60 \%)$ patients were included into the typical group while 49 (40\%) into the atypical group. No significant differences in gender or number of comorbidities were noted between the two groups. However, a larger proportion of patients with dementia $(33 \%$ vs $21 \%, P=0.13)$ presented with atypical symptoms.

In the atypical group, the most common presenting complaint was fall in 18 patients (36\%), reduced mobility or generalised weakness in $18(36 \%)$ and delirium in $11(22 \%)$. 
Table 1 Comparison of patient demographics and characteristics

\begin{tabular}{|c|c|c|c|c|c|c|c|}
\hline \multirow[b]{3}{*}{ Age } & \multicolumn{2}{|l|}{ All } & \multicolumn{2}{|l|}{ Typical } & \multicolumn{2}{|c|}{ Atypical } & \multirow[t]{2}{*}{$P$ value } \\
\hline & \multicolumn{2}{|c|}{$n=122$} & \multicolumn{2}{|l|}{$n=73$} & \multicolumn{2}{|c|}{$n=49$} & \\
\hline & \multicolumn{3}{|c|}{$\%$} & $\%$ & & $\%$ & \\
\hline Mean \pm SD & \multicolumn{2}{|c|}{$81 \pm 8$} & \multicolumn{2}{|l|}{$81 \pm 8$} & \multicolumn{2}{|c|}{$81 \pm 8$} & 0.69 \\
\hline $65-74$ & 27 & 22 & 17 & 23 & 10 & 20 & \\
\hline $75-84$ & 50 & 41 & 32 & 44 & 18 & 37 & \\
\hline$\geq 85$ & 45 & 37 & 24 & 33 & 21 & 43 & \\
\hline Sex & & & & & & & 0.97 \\
\hline Male & 62 & 51 & 37 & 51 & 25 & 51 & \\
\hline Female & 60 & 49 & 36 & 49 & 24 & 49 & \\
\hline \multicolumn{8}{|l|}{ COVID-19 diagnosis } \\
\hline Swab & 106 & 87 & 62 & 85 & 44 & 90 & \\
\hline Radiological & 16 & 13 & 11 & 15 & 5 & 10 & \\
\hline \multicolumn{8}{|l|}{ Comorbidities } \\
\hline Dementia & 31.0 & 25 & 15 & 21 & 16 & 33 & 0.13 \\
\hline Hypertension & 69.0 & 57 & 41 & 56 & 28 & 57 & 0.92 \\
\hline Coronary artery disease & 26.0 & 21 & 17 & 23 & 9 & 18 & 0.52 \\
\hline Congestive heart failure & 20.0 & 16 & 14 & 19 & 6 & 12 & 0.31 \\
\hline COPD/asthma & 25.0 & 20 & 16 & 22 & 9 & 18 & 0.63 \\
\hline $\mathrm{T} 2 \mathrm{DM}$ & 43.0 & 35 & 26 & 36 & 17 & 35 & 0.92 \\
\hline CKD & 27.0 & 22 & 14 & 19 & 13 & 27 & 0.34 \\
\hline On immunosuppression & 11.0 & 9 & 6 & 8 & 5 & 10 & 0.71 \\
\hline Pre-existing DNAR decision & 33 & 27 & 20 & 27 & 13 & 27 & \\
\hline \multicolumn{8}{|l|}{ Number of comorbidities } \\
\hline 0 & 4 & 3 & 3 & 4 & 1 & 2 & 0.53 \\
\hline 1 & 17 & 14 & 11 & 15 & 6 & 12 & 0.66 \\
\hline 2 & 22 & 18 & 14 & 19 & 8 & 16 & 0.69 \\
\hline 3 & 26 & 21 & 13 & 18 & 13 & 27 & 0.25 \\
\hline$\geq 4$ & 53 & 43 & 32 & 44 & 21 & 43 & 0.92 \\
\hline Residence & & & & & & & 0.27 \\
\hline Home & 89.0 & 72 & 50 & 69 & 38 & 78 & \\
\hline Care institution & 34.0 & 28 & 23 & 31 & 11 & 22 & \\
\hline Clinical Frailty Score & & & $n=72$ & & & & \\
\hline$<3$ & 33 & 27 & 24 & 33 & 9 & 18 & 0.07 \\
\hline 4 & 13 & 11 & 8 & 11 & 5 & 10 & 0.87 \\
\hline$\geq 5$ & 75 & 61 & 40 & 56 & 35 & 71 & 0.08 \\
\hline
\end{tabular}

$S D$ standard deviation, $C O P D$ chronic obstructive pulmonary disease, $T 2 D M$ type 2 diabetes mellitus, $C K D$ chronic kidney disease, DNAR do not attempt resuscitation

Further assessment by paramedics and in the emergency department (ED) found $32(65 \%)$ to have underlying typical features of COVID-19, fever being the most common $(n=$ $29,91 \%)$, followed by cough $(n=6,19 \%)$ and shortness of breath $(n=4,13 \%)$. This subset of patients had worse outcomes than those in the typical group with a mortality rate of $50 \%$ versus $38 \%$, respectively, although this was not statistically significantly $(P=0.23)$. Vital observation on attendance to the ED found 22 (45\%) to be hypoxic, 15 (31\%) to be tachycardic and $11(22 \%)$ to be tachypnoeic.

In the atypical group, 16 patients (33\%) had consultations with their primary care practitioner prior to admission to hospital. Eleven (69\%) were subsequently commenced on oral antibiotics ( 9 for urinary tract infection (UTI) and 2 for chest infection) with no improvement. The median duration of antibiotic treatment before presenting to hospital was 3 days (IQR 1-7).

Thiry-five (71\%) patients in the atypical group scored $\geq 5$ on the Rockwood CFS compared with $40(56 \%)$ in the typical group, although no statistical significance was recorded $(P=$ $0.08)$. There was also no significant difference in the COVID19 laboratory workup with lymphopenia and elevated CRP, D-dimer, LDH, troponin T and CK demonstrated in both groups. The comparative findings between the atypical and typical groups are summarised in Table 2.

Four patients (6\%) in the typical group required intubation and ventilation compared with $1(2 \%)$ in the atypical group $(P$ $=0.76$ ). Continuous positive airway pressure (CPAP) support was required in $3(4 \%)$ patients in the typical group and $3(6 \%)$ patients in the atypical group. Three patients (43\%) in the typical group and 4 patients $(100 \%)$ in the atypical group who required ventilatory support subsequently died $(P=$ $0.06)$. There were no significant differences in overall mortality or length of hospital stay between the two groups (Table 2). A total of 28 deaths (38\%) and 17 deaths (35\%) were reported in the typical and atypical groups, respectively $(P=0.68)$.

\section{Discussion}

Our study demonstrated that 40\% (49/122) of our older population with COVID-19 had atypical presenting complaints in the community. Although patients with atypical presentation appeared to have worse outcomes, there was no statistically significant difference between the atypical and the typical groups. Therefore, we suggest that it may not be possible to prognosticate patients based on presenting symptoms. The rate of atypical presentations of COVID-19 observed was similar to that found by Hofman and colleagues in 2017 for a general medical patient cohort aged $\geq 80$ years presenting to an emergency department. The most common atypical presentation reported was "fall", also demonstrated in our study, and frequent underlying diagnoses were found to be infections, neurological insults and fractures. This suggests that the physiological response of older adults towards COVID-19 may be parallel to that seen in other disease processes [6].

In acute illness states, older adults respond differently to young adults. Although aetiologies may be multifactorial, differences in physiological and immune responses are likely important contributors to varied presentation. Diminished central thermoregulation by the hypothalamus and the lack of 
Table 2 Comparison of main presenting complaints, vital signs on arrival to ED, laboratory investigations and clinical outcome

\begin{tabular}{|c|c|c|c|c|c|}
\hline & \multicolumn{2}{|l|}{ Typical } & \multicolumn{2}{|l|}{ Atypical } & \multirow[t]{2}{*}{$P$ value } \\
\hline & \multicolumn{2}{|l|}{$n=73$} & \multicolumn{2}{|l|}{$n=49$} & \\
\hline Signs and symptoms & & $\%$ & & $\%$ & \\
\hline Cough & 28 & 38 & & & \\
\hline Fever & 22 & 30 & & & \\
\hline Dyspnoea & 53 & 73 & & & \\
\hline Confusion & 8 & 11 & 11 & 22 & \\
\hline Reduced GCS & 1 & 1 & 6 & 12 & \\
\hline Fall/collapse & 1 & 1 & 18 & 37 & \\
\hline Off legs/generalised weakness & 3 & 4 & 18 & 37 & \\
\hline Reduced oral intake & 4 & 6 & 7 & 14 & \\
\hline \multicolumn{6}{|l|}{ Others } \\
\hline Diarrhoea & 4 & 6 & 2 & 4 & \\
\hline Chest pain & & & 1 & 2 & \\
\hline Headache & 1 & 1 & & & \\
\hline Sore throat & 1 & 1 & & & \\
\hline Vomiting & & & 1 & 2 & \\
\hline \multicolumn{6}{|l|}{ Vital signs on arrival in ED } \\
\hline Heart rate (beats per minute) & \multicolumn{2}{|c|}{93 (IQR 82-109, range 40-144) } & \multicolumn{2}{|c|}{89 (IQR 80-105; range 63-149) } & 0.55 \\
\hline Respiratory rate (breaths per minute) & \multicolumn{2}{|c|}{24 (IQR 22-28, range 15-33) } & \multicolumn{2}{|c|}{20 (IQR 18-24; range 11-46) } & $<0.05^{*}$ \\
\hline Mean arterial pressure $(\mathrm{mmHg})$ & \multicolumn{2}{|c|}{97 (IQR 86-110, range 58-139) } & \multicolumn{2}{|c|}{94 (IQR 78-107; range 64-139) } & 0.07 \\
\hline Hypoxia & 48 & 66 & 22 & 45 & $0.02 *$ \\
\hline \multicolumn{6}{|l|}{ Laboratory findings } \\
\hline Leucocytes $\left(\times 10^{9} / \mathrm{L}\right)$ & \multicolumn{2}{|l|}{7.50 (IQR 5.67-9.05) } & \multicolumn{2}{|l|}{7.48 (IQR 5.67-9.66) } & 0.47 \\
\hline Neutrophils $\left(\times 10^{9} / \mathrm{L}\right)$ & \multicolumn{2}{|l|}{5.52 (IQR 3.90-7.49) } & \multicolumn{2}{|l|}{5.40 (IQR 4.10-8.12) } & 0.56 \\
\hline Lymphocytes $\left(\times 10^{9} / \mathrm{L}\right)$ & \multicolumn{2}{|l|}{0.89 (IQR 0.61-1.33) } & \multicolumn{2}{|l|}{$0.82($ IQR $0.65-1.20)$} & 0.47 \\
\hline C-reactive Protein $(\mathrm{mg} / \mathrm{L})$ & \multicolumn{2}{|l|}{76 (IQR 42.5-144) } & \multicolumn{2}{|l|}{76.0 (IQR 36.5-134.5) } & 0.83 \\
\hline Urea $(\mathrm{mmol} / \mathrm{L})$ & \multicolumn{2}{|l|}{7.90 (IQR 6.2-11.9) } & \multicolumn{2}{|l|}{8.3 (IQR 6.75-15.5) } & 0.23 \\
\hline Creatinine $(\mu \mathrm{mol} / \mathrm{L})$ & \multicolumn{2}{|l|}{91 (IQR 71-124) } & \multicolumn{2}{|l|}{104 (IQR 76-152) } & 0.21 \\
\hline D-dimer (ng/mL) & 1295 (IQR 898-3430) & $n=20$ & 1917 (IQR 755-3483) & $n=13$ & 0.90 \\
\hline Lactate dehydrogenase (IU/L) & 420 (IQR 259-520) & $n=30$ & 305 (IQR 236-410) & $n=18$ & 0.08 \\
\hline Troponin (ng/L) & 29 (IQR 17-53) & $n=38$ & 35 (IQR 23-42) & $n=19$ & 0.77 \\
\hline Creatine kinase $(\mu \mathrm{mol} / \mathrm{L})$ & 211 (IQR 45-664) & $n=21$ & 122 (IQR 60-974) & $n=15$ & 0.59 \\
\hline Acute kidney injury & 23 & 32 & 19 & 39 & 0.41 \\
\hline Clinical outcome & & & & & \\
\hline Ventilatory support & 7 & 10 & 4 & 8 & 0.79 \\
\hline Intubation & 4 & 6 & 1 & 2 & \\
\hline CPAP & 3 & 4 & 3 & 6 & \\
\hline Mortality rate in patients who required ventilatory support & 3 & 43 & 4 & 100 & 0.06 \\
\hline Total In-hospital mortality & 28 & 38 & 17 & 35 & 0.68 \\
\hline Length of stay & 14 (IQR 9-20; range 1 & & $16^{\dagger}$ (IQR 11-18; range & & 0.50 \\
\hline
\end{tabular}

GCS Glasgow Coma Scale, ED emergency department, CPAP continuous positive airway pressure, IQR interquartile range. ${ }^{\dagger} 1$ patient in the atypical group is still an inpatient

response to endogenous cytokines such as interleukin (IL) 1 and 6 related to ageing may explain the absence of pyrexia in the presence of infection [9]. The pathophysiology of delirium may also occur in a similar fashion where central hormonal instabilities occur, particularly in the presence of acute stress and inflammation. Patients with ageing and central nervous system diseases such as cerebrovascular or Alzheimer's dementia are therefore highly vulnerable to delirious states 
which can complicate the diagnosis of acute illness in hospital [10]. This may explain the increased numbers of patients with underlying dementia and high premorbid clinical frailty scores seen in our atypical presentation group.

Furthermore, it has been suggested that immune responses are dysregulated in an ageing physiology which may account for the higher incidence of severe forms of COVID-19 observed in older adults [11]. The hallmark of COVID-19 is the cytokine "storm" syndrome where a surge in proinflammatory cytokines causes endothelial breakdown and an increase in vascular permeability. This in turn leads to fluid entry into the alveoli causing hypoxia and, in severe cases, acute respiratory distress syndrome [12, 13]. Cited markers of cytokine release were elevated in both groups and concomitant "silent hypoxia" was prominent in our atypical cohortextrapolated physiological implications of these factors may explain the high number of deaths reported in nursing and care homes. Notably, we have demonstrated that this group of patients, who initially present atypically, but are later found to have typical symptoms of COVID-19 such as pyrexia and dyspnoea with concomitant hypoxia, tend to have a worse clinical outcome which could be attributed to subsequent respiratory failure and/or $\geq 2$ organ failure. While it would be interesting to confirm this in a larger prospective study, we postulate that early oxygenation and supportive therapy may improve survival. Moreover, it has been demonstrated that older adults are prone to bacterial superinfection and empirical treatment with antibiotics may be justified [4].

Increasing institutionalisation and the number of older adults living alone with little to no social support may be an additional contributing factor to the death rates seen in the UK. Lockdown measures imposed may have potentially delayed appropriate treatment in secondary care for two main reasons: firstly, there was fear among elderly patients of contracting COVID-19 in hospital, and secondly, there were huge pitfalls and challenges to diagnosis with telephone consultations (the primary care point of contact) without formal clinical assessment. Furthermore, we noted that a subset of patients initially treated for UTI or chest infection by general practitioners subsequently presented to hospital with worsening symptoms. We therefore urge clinicians, particularly in primary care, to have a low threshold for SARS-CoV-2 testing in older patients and to consider prompt referral to hospital if symptoms are non-resolving. These measures would also positively impact on community and institutional nosocomial transmission.

At the time of our study, the National Institute of Clinical Excellence (NICE) committee had initiated guidelines for critical care resource allocation in a pandemic state. For patients with a CFS $\geq 5$ (at the discretion of treating clinicians), ventilatory support other than oxygen administration and escalation to intensive care units was thought inappropriate because of poor outcomes [14]. We recognise that this may have underestimated both the severity of disease in each group and the number of patients who would have been provided full escalation of care in normal circumstances. Nonetheless, a comparison of mortality rates in both groups yielded similar results. In light of this, patients with atypical presentations of the illness should be given the same attention (arguably more) as those who present with typical symptoms.

RT-PCR testing has a theoretical sensitivity of up to $95 \%$ [15] which is comparable with the results seen in our study with $87 \%$ of our patients testing positive. However, in clinical practice, it has been reported that the sensitivity of the test reaches only $60-70 \%$. Because the reliability of the RT-PCR test is dependent on specimen quality and timing, a negative test is insufficient to exclude COVID-19, and in suspected cases a radiological diagnosis may provide better sensitivity [16]; this is relevant when high clinical suspicion persists within the context of negative RT-PCR result. The nasopharyngeal swab for SARS-CoV-2 should be repeated if clinical suspicion remains strong.

\section{Limitations}

Our study should be viewed in the context of certain limitations. As a single-centre study at a district general hospital, we cannot generalize our results to other practice settings. Furthermore, our trust is based in the south of England, and demographics and socioeconomic status of our population may differ from those elsewhere.

We recognise the retrospective nature of our study and small sample size as limitations. We also acknowledge that extracting information from medical notes requires secondhand interpretation and may not be representative of the full clinical picture. Patient outcomes may also be influenced by the guidelines imposed by the NICE committee as previously mentioned [14]. As our study only evaluated hospitalised patients, it may not accurately reflect the true prevalence of atypical symptoms among older adults in the community. We recommend further studies with larger sample sizes be conducted to validate whether early detection, observant monitoring and hospital admission improves outcomes.

\section{Conclusion}

As lockdown measures ease globally, older adults remain inherently vulnerable to COVID-19. Our results clearly demonstrate that approximately $40 \%$ of this population present with atypical symptoms of COVID-19 which include falls, reduced mobility, weakness and confusion as the principal complaint in the community. These patients are equally and possibly more susceptible to poor outcomes and subsequent death. Emphasis is laid on the urgency to increase nationwide testing capacity because early detection is likely to improve prognosis 
and retard community transmission, especially within institutions. The importance of comprehensive clinical assessment of elderly patients on presentation to hospital, expectant observation thereafter and appropriate early intervention are underscored. Primary care practitioners need to remain vigilant for subclinical, subtle or non-specific symptoms of COVID-19 and should have a low threshold for onward referral to secondary care.

Authors' contributions All authors contributed to the study conception and design. Material preparation, data collection and analysis were performed by JG, JK, MAF and HMC. The first draft of the manuscript was written by JG and JK, and all authors commented on previous versions of the manuscript. AM, RC and $\mathrm{CM}$ edited the manuscript up to submission. All authors read and approved the final manuscript.

\section{Compliance with ethical standards}

Conflict of interest The authors declare that they have no conflict of interest.

Ethical approval This survey was approved by the trust audit department with reference FH125. As a registry report using clinically collected, non-identifiable data, this work does not fall under the remit of NHS Research Ethics Committees. All procedures performed in studies involving human participants were in accordance with the ethical standards of the institutional and/or national research committee and with the 1964 Helsinki declaration and its later amendments or comparable ethical standards.

\section{References}

1. Gov.uk (2020) Coronavirus (COVID-19) in the UK https:// coronavirus.data.gov.uk. Accessed 11 June 2020

2. Holroyd-Leduc J, Gandell D, Miller A, Petrov D (2020) COVID-19 in older adults https://www.rgptoronto.ca/wp-content/uploads/ 2020/04/COVID-19-Presentations-in-Frail-Older-Adults-U-of-Cand-U-fo-T.pdf. Accessed 11 June 2020

3. Guan WJ, Ni ZY, Hu Y et al (2020) Clinical characteristics of coronavirus disease 2019 in China. N Engl J Med 382(18):17081720 https:/www.nejm.org/doi/full/10.1056/NEJMoa2002032

4. Liu K, Chen Y, Lin R, Han K (2020) Clinical features of COVID19 in elderly patients: a comparison with young and middle-aged patients. J Infect 80(6):e14-e18. https://doi.org/10.1016/j.jinf.2020. 03.005

5. Zhou F, Yu T, Du R et al (2020) Clinical course and risk factors for mortality of adult inpatients with COVID-19 in Wuhan, China: a retrospective cohort study. Lancet 395(10229):1054-1062. https:// doi.org/10.1016/S0140-6736(20)30566-3

6. Hofman MR, van den Hanenberg F, Sierevelt IN, Tulner CR (2017) Elderly patients with an atypical presentation of illness in the emergency department. Neth J Med 75(6):241-246

7. Burki T (2020) England and Wales see 20000 excess deaths in care homes. Lancet 395(10237):1602-6736(20)31199-5. https:// doi.org/10.1016/S0140-6736(20)31199-5

8. Solanki T (2020) Atypical Covid-19 presentations in older peoplethe need for continued vigilance. https://www.bgs.org.uk/blog/ atypical-covid-19-presentations-in-older-people-\%E2\%80\%93the-need-for-continued-vigilance. Accessed 11 June 2020

9. Norman DC (2000) Fever in the elderly. Clin Infect Dis 31(1):148 151. https://doi.org/10.1086/313896

10. Maclullich AM, Ferguson KJ, Miller T et al (2008) Unravelling the pathophysiology of delirium: a focus on the role of aberrant stress responses. J Psychosom Res 65(3):229-238. https://doi.org/10. 1016/j.jpsychores.2008.05.019

11. Fuentes E, Fuentes M, Alarcón M, Palomo I (2017) Immune system dysfunction in the elderly. An Acad Bras Cienc 89(1):285-299. https://doi.org/10.1590/0001-3765201720160487

12. Brune K, Frank J, Schwingshackl A et al (2015) Pulmonary epithelial barrier function: some new players and mechanisms. Am J Physiol Lung Cell Mol Physiol 308(8):L731-L745. https://doi. org/10.1152/ajplung.00309.2014

13. Mehta P, McAuley DF, Brown M et al (2020) COVID-19: consider cytokine storm syndromes and immunosuppression. Lancet 395(10229):1033-1034. https://doi.org/10.1016/s0140-6736(20) 30628-0

14. National Institute of Clinical Excellence (NICE) (2020) COVID-19 rapid guideline: critical care in adults. https://www.nice.org.uk/ guidance/ng159/resources/critical-care-admission-algorithm-pdf8708948893. Accessed 11 June 2020

15. Corman VM, Landt O, Kaiser M et al (2020) Detection of 2019 novel coronavirus $(2019-\mathrm{nCoV})$ by real-time RT-PCR. Euro Surveill 25(3):2000045. https://doi.org/10.2807/1560-7917.ES. 2020.25.3.2000045

16. Ai T, Yang Z, Hou H et al (2020) Correlation of chest CT and RTPCR testing in coronavirus disease 2019 (COVID-19) in China: a report of 1014 cases. Radiology 200642:E32-E40. https://doi.org/ $10.1148 /$ radiol.2020200642

Publisher's note Springer Nature remains neutral with regard to jurisdictional claims in published maps and institutional affiliations. 